\title{
Antidepressant and Proneurogenic Influence of Environmental Enrichment in Mice: Protective Effects vs Recovery
}

\author{
María Llorens-Martín', Gonzalo S Tejeda' and José L Trejo*,' \\ 'Department of Molecular, Cellular and Developmental Neurobiology, Cajal Institute, CSIC, Madrid, Spain
}

Physical-cognitive activity has long-lasting beneficial effects on the brain and on behavior. Environmental enrichment (EE) induces brain activity known to influence the behavior of mice, as measured in learned helplessness paradigms (forced swim test), and neurogenic cell populations in the hippocampal dentate gyrus. However, it is not completely clear whether the antidepressant and proneurogenic effects of EE are different in animals that are naive or pre-exposed to the stress inducing helplessness, and if this depends on the type of stressor. It also remains unclear whether differential effects are exerted on distinct neurogenic subpopulations. We found that EE has a protective effect in adult female mice (C57BL/6) when exposed twice to the same stressor (forced swim test) but it has no influence on recovery. The repeated exposure to this stressor was analyzed together with the effects of EE on different neurogenic populations distinguished by age and differentiation state. Younger cells are more sensitive and responsive to the conditions, both the positive and negative effects. These results are relevant to identify the cell populations that are the targets of stress, depression, and enrichment, and that form part of the mechanism responsible for mood dysfunctions.

Neuropsychopharmacology (20II) 36, 2460-2468; doi:I0.1038/npp.20 II.134; published online 27 July 20I I

Keywords: environmental enrichment; adult hippocampal neurogenesis; learned helplessness; Porsolt test; doublecortin; cell survival

\section{INTRODUCTION}

Physical-cognitive activity and sedentary lifestyle are two opposing paradigms that are fundamental to understand how some brain functions crucial to behavior are regulated. We mean by physical-cognitive activity in murine models, the physical exercise and the processing of information (novel and much higher than normally processed by control animals) induced by environmental enrichment (EE).

These paradigms influence neural plasticity (Cordoba et al, 1984; García-Segura, 2009; Nithianantharajah and Hannan, 2009; Petrosini et al, 2009; Sale et al, 2009) and synaptic plasticity (Christie et al, 2008), inducing changes in the rate of adult hippocampal neurogenesis (Kempermann et al, 1997b). Moreover, in the long-term, such activity has a direct impact on the ability to cope with life events, known as animal's cognitive reserve (Mandolesi et al, 2008; Milgram et al, 2006) and neurogenic reserve (Kempermann, 2008), respectively. Indeed, these contrasting paradigms influence the individual's ability to cope with

*Correspondence: Dr JL Trejo, Department of Molecular, Cellular and Developmental Neurobiology, Cajal Institute, CSIC, Av. Doctor Arce 37, 28002 Madrid, Spain, Tel: + 3491585 465I, Fax: + 34 91 585 4754, E-mail: jitrejo@cajal.csic.es

Received 9 February 201 I; revised 16 June 2011; accepted 20 June 2011 stress and depression (Greer and Trivedi, 2009; Laviola et al, 2008; Mora et al, 2007), specifically in relation to their effects on adult neurogenesis (Navailles et al, 2008; Schloesser et al, 2010). We now know that EE augments neurogenesis by specifically stimulating either the survival or proliferation of newborn neurons (Kempermann et al, 1997a, b, 1998; Olson et al, 2006; van Praag et al, 1999). In addition, it was recently shown that specific, non-indiscriminate effects of EE may be channeled through specific subpopulations of immature newborn neurons (LlorensMartin et al, 2007, 2010). However, there is also evidence of the beneficial effects of enrichment in the absence of adult hippocampal neurogenesis (Meshi et al, 2006).

The details of the antidepressant and proneurogenic effects of EE have been studied in different animal and disease models (eg see Leal-Galicia et al, 2007; Navailles et al, 2008; Schaeffer et al, 2009; Segovia et al, 2006; Sztainberg et al, 2010). However, there is still much to learn about the effects of EE in previously depressed and/or stressed animals, as well as the effect of pre-exposure to the same stressor. This is true both at the behavioral level and in terms of the morphological changes in the brain including the effects on adult hippocampal neurogenesis. Thus, we were interested in determining if the antidepressant effect of EE, as measured in a Porsolt test of forced swimming (learned helplessness) after a long time period of $\mathrm{EE}$, is equivalent in naive animals and in animals pre-exposed to the same Porsolt test before the start of EE. 
We hypothesized that the effect of pre-exposure will depend on the type of helplessness-inducing stressor used, and that the effects will be different from other published models of stress like social conflict (Schloesser et al, 2010). In addition, we assessed whether this experimental model has different effects on two adult subpopulations that undergo neurogenesis by labeling immature neurons at two different ages and stages of cell differentiation. Our hypothesis was that different cell populations will respond distinctly to these manipulations, as previous studies suggest that not all cells respond uniformly to EE (Llorens-Martin et al, 2007, 2010). By labeling these cells in the same animal with immunohistochemically compatible and distinguishable thymidine analogs, we also assessed whether there was any quantitative relationship between these subpopulations. We hypothesized that younger neurons would be more capable of reacting to both the protective and deleterious effects of the EE and stress.

Several behaviors like hippocampal-dependent learning and memory, anxiety, and depression-like behavior have recently been evaluated to assess indirect or direct relationship to adult hippocampal neurogenesis, as a possible mechanism mediating the effects of $\mathrm{EE}$, physical exercise, and other interventions (for a recent review see, eg, Llorens-Martin et al, 2009). Both neurogenesis-dependent and -independent mechanisms have been demonstrated to be involved in these effects (Trejo et al, 2008). In the present work, we have tried to gain further evidence about whether hippocampal newborn neurons are regulated as expected if they are involved in the effects of EE on the depression-like behavior.

In addition, we also wanted to determine whether $\mathrm{EE}$ sensitizes the brain to novelty (which might influence its anxiolytic effect; see, eg, Fox et al, 2006; Stead et al, 2006) or by contrast, if it desensitizes the brain to novelty, so that the animal might develop a higher threshold to the perception of threat in the environment (which could lead to learn helplessness paradigms with a different temporal pattern, a putative behavioral correlate of the antidepressant effect of $\mathrm{EE})$. To this end, animals were subjected to EE before the Porsolt test, after a homotypic pre-exposure to the same stressor (Porsolt test). Pre-exposure to forced swimming is associated with sensitization to the subsequent exposure (a lower threshold), and this effect may persist for months. We hypothesize that the effect of EE is not dependent on desensitization to novelty and, therefore, EE should have no effect on pre-exposed animals in our paradigm.

By addressing these issues, we aim to identify target cell populations involved in stress, depression, and enrichment that may also participate in the dysfunctions of mood states in animal models.

\section{MATERIALS AND METHODS}

\section{Animals}

Adult C57/BL6J female mice, 8 weeks of age $(n=32$, Harlan Laboratories) were housed at $22 \pm 1{ }^{\circ} \mathrm{C}$ on a 12/12-h light/ dark cycle, and with ad libitum access to food and water. Mice were kept under standard laboratory conditions in accordance with the European Community Guidelines (directive 86/609/EEC).

\section{Experimental Design}

After a quarantine of 1 week, mice were randomly distributed into four experimental groups (Figure 1). Accordingly, 16 female mice were exposed to a EE protocol for 10 weeks, in order to compare them with 16 control mice housed under standard laboratory conditions (SHC). From each of these groups, eight mice were subjected to a Porsolt forced swim test (CPP and EPP groups) immediately before the beginning of the EE protocol, whereas the other eight mice from each group were not exposed to this first Porsolt forced swim test (CNP and ENP groups). Finally, all the mice were subjected to a second forced swim test at the end of the experiment to determine how environmental richness affects the depressive-like behavior of mice, previously subjected or not, to such an acute highly stressful situation.

\section{CldU and IdU Injection Protocol}

We used thymidine analogs at equimolecular dosages to BrdU administration. With this single goal, equimolecular doses to $50 \mathrm{mg} / \mathrm{kg} \mathrm{BrdU}$ for both CldU (5-chloro-2'-deoxyuridine) and IdU (5-iodo-2'-deoxy-uridine) were calculated as published elsewhere (Llorens-Martin et al, 2010), and accordingly to the findings recently published (Dupret et al, 2008; Leuner et al, 2009).

All mice received one daily injection of CldU (i.p. $57.65 \mathrm{mg} / \mathrm{kg}$ bw) injection per day during the first 4 days of the experiment, and one daily injection of IdU (i.p. $42.75 \mathrm{mg} / \mathrm{kg} \mathrm{bw}$ ) on 4 consecutive days during the last week of the EE protocol.

\section{Environmental Enrichment}

We used an EE protocol (Hutchinson et al, 2005) involving classical toys and objects, as well as a running wheel (Martinez-Cue et al, 2002). This paradigm has proven useful to modulate some aspects of adult hippocampal

a

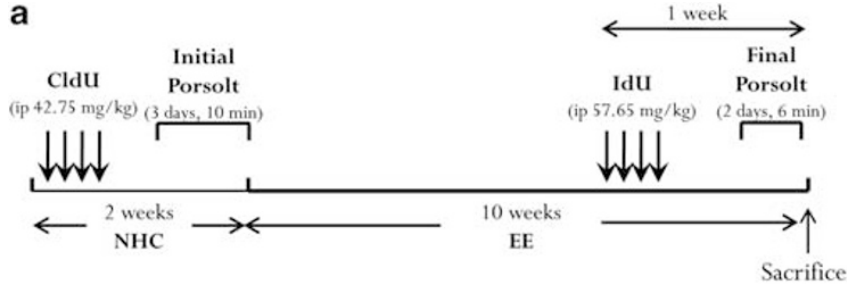

\begin{tabular}{|l|c|c|c|}
\hline $\begin{array}{c}\text { Experimental } \\
\text { Group }\end{array}$ & $\begin{array}{c}\text { Initial } \\
\text { Porsolt }\end{array}$ & Housing conditions & $\begin{array}{c}\text { Final } \\
\text { Porsolt }\end{array}$ \\
\hline$C N P$ & No & Normal Housing & Yes \\
\hline$C P P$ & Yes & Normal Housing & Yes \\
\hline$E N P$ & No & Enriched environment & Yes \\
\hline$E P P$ & Yes & Enriched environment & Yes \\
\hline
\end{tabular}

Figure I Experimental design. (a) Diagram of the experimental plan. Animals received CldU injections on 4 consecutive days at the beginning of the experiment. The following week, half the animals were exposed to an initial Porsolt test during 3 consecutive days. Immediately afterwards, the animals were housed either in normal cages or in an enriched environment for 10 weeks. One week before the end of the experiment, they received IdU injections on 4 consecutive days. Two days before killing, all the animals were exposed to a final Porsolt test. (b) Table with the four experimental groups resulting from the combinations of the different treatments. 
neurogenesis, as indicated previously (Llorens-Martin et al, 2007, 2010).

\section{Porsolt Test}

We used the Porsolt test to measure depressive-like behavior, and as a stress model (Porsolt et al, 1977a,b). Briefly, animals were placed in a $12-\mathrm{cm}$ diameter and $29 \mathrm{~cm}$ tall cylinder filled with $23{ }^{\circ} \mathrm{C}$ water 2 (or 3 ) consecutive days for $6 \mathrm{~min}$ each day. First Porsolt test consisted on three consecutive days in order to asses a hard depressive-like status of mice that performed the first forced swim test (CPP and EPP groups) compared with naive animals (CNP and ENP groups). However, the duration of the second Porsolt test was a standard one (2 days). Performance of animals were recorded with a camera (JVC Mod QZ MG 20), transferring videoclips directly to a hard disk. The behaviors were assigned to different categories, according to Detke et al (1995) and Llorens-Martin et al (2007). Data are presented as time of immobility plus staying afloat in the second day.

\section{Histology}

Twenty-four hours after the second Porsolt test had been completed, the mice were deeply anesthetized by an intraperitoneal penthobarbital injection (EutaLender, $60 \mathrm{mg} / \mathrm{kg} \mathrm{bw}$ ) and they were then transcardially perfused with saline. The animals' brain was removed and post-fixed overnight with $4 \%$ paraformaldehyde in phosphate buffer.

Coronal sections of the brains were obtained on a Leica VT1000S vibratome ( $50 \mu \mathrm{m}$ thick sections). The serial vibratome sections of the hippocampal formation were collected individually in 96-multiwell culture plates. Series of brain slices were obtained from one section in every nine to perform immunohistochemistry. Randomly chosen series was Nissl stained to calculate the total volume of each animal's dentate gyrus.

The sections were initially pre-incubated in $\mathrm{PB}$ with Triton X-100 0.5\% and bovine serum albumin $0.1 \%$, and then dual immunohistochemistry was performed as described previously (Llorens-Martin et al, 2006). The following primary antibodies were utilized: a rat anti-CldU antibody (Accuratechemicals, 1:500), a mouse anti-IdU antibody (BD Biosciences, 1:500), a rabbit anti-phosphohistone3 antibody (Upstate, 1:500), a rabbit anti-Ki67 antibody (ThermoScientific, 1:750), a goat anti-doublecortin (anti-DCX) antibody (Santa Cruz, 1:500), a rabbit anticalretinin (anti-Calret) antibody (Swant, 1:3000); a guinea pig anti-vesicular glutamate transporter1 (vGlut1) (Chemicon, 1:2500), a mouse anti-glutamic acid decarboxylase (GAD) (Developmental Hybridome Bank, 1:500), a rabbit anti-cfos antibody (Calbiochem, 1:10000), and a rabbit anti-fractin antibody (BD Biosciences, 1:500; fractin is an actin fragment cleaved by caspase). Secondary Alexa-labeled antibodies from Molecular Probes were used at a final concentration of $1: 1000$. The following antibodies were used: donkey anti-rabbit 594 Alexa-conjugated for the anti-phosphohistone3 antibody detection, donkey anti-rabbit 568 Alexa-conjugated for the anti-cfos antibody detection, donkey anti-rat 488 Alexa-conjugated for the anti-CldU antibody detection, donkey anti-mouse 594
Alexa-conjugated for the anti-IdU and anti-GAD antibodies detection, donkey anti-goat 594 Alexa-conjugated for the anti-DCX antibody detection, donkey anti-rabbit 488 Alexaconjugated for the anti-Calret antibody detection, and goat anti-guinea pig biotin-conjugated for the anti-vGLUT1 antibody detection followed by incubation with a 488 Streptavidin Alexa-conjugated. All sections were counterstained with DAPI (Calbiochem, 1:1000). The incubation periods used ranged from 24 to $48 \mathrm{~h}$ at $4{ }^{\circ} \mathrm{C}$ for primary antibodies, $24 \mathrm{~h}$ at $4{ }^{\circ} \mathrm{C}$ for secondary antibodies, and $10 \mathrm{~min}$ for DAPI incubation.

We have used both phosphohistone 3 and Ki67 antibodies to label cell proliferation. Phosphohistone3 antibody labels cells in mitosis, and Ki67 antibody labels cells running through any point of the cell cycle. Fractin has been used to measure cell death. Fractin antibody labels actin fragments cleaved by caspase. To obtain the percentage of either $\mathrm{IdU}^{+}$ cells or CldU ${ }^{+}$cells double labeled by DCX, $100 \mathrm{IdU}^{+}$cells, and $100 \mathrm{CldU}^{+}$cells per animal were checked for colabeling by using the confocal microscope mentioned above.

\section{Cell Counting}

The total number of phosphohistone3, cfos, CldU, and IdU-positive cells were counted using the optical dissector method as previously described (Llorens-Martin et al, 2006) and an optical fluorescence microscope (Leica DMI 6000 B, oil immersion $\times 40$ objective). The total number of immature DCX and Calret-positive cells and of mature granule neurons was analyzed by applying a physical dissector method developed for the confocal microscope (Leica TCS SP5) as described previously (Llorens-Martin et al, 2006). The total area occupied by either glutamatergic or gabaergic terminals in the inner molecular layer was obtained as described elsewhere (Trejo et al, 2007) and measured with the help of semiautomatic image analysis software (ImageJ, v. 1.33, NIH, Bethesda, MD, USA; http:// rsb.info.nih.gov/ij). This same software was used to count the total number of immature and mature granule neurons.

\section{Statistical Analysis}

The cell numbers and mouse behavior was analyzed by applying a two-way ANOVA test to compare the data from the four experimental groups. The Tukey test was also carried out for post hoc comparisons between paired groups. Correlations between different cell subpopulations were analyzed by the bivariate correlation test. SPSS 17.0.1 software (SPSS, 1989; Apache Software Foundation) was used to analyze all the data.

\section{RESULTS}

The experimental design established here was intended to determine the effect of exposing adult female mice to a Porsolt test in terms of behavior and adult hippocampal neurogenesis. These effects were assessed both in naive mice and in mice that had been previously subjected to the same stressor, both in animals that experienced EE or in those maintained under normal housing conditions (Figure 1).

We found that the different groups performed differently in the forced swim test (two-way ANOVA for housing and 
Porsolt; ANOVA F 3,25 26.513, $p<0.0001$. F housing 1,25 18.729, $p=0.0001 ; F_{\text {Porsolt } 1,25} 45.022, p=0.0001 ; F_{\text {housing } \times \text { Porsolt 1, } 25}$ 11.216, $p=0.003)$. A 10 -week period of $\mathrm{EE}$ induced a significant and pronounced decrease in the time mice remained immobile (ENP) in the Porsolt test after enrichment (the so-called final Porsolt) when compared with the control animals (CNP; Figure 2). This effect was only evident in naive animals that had not previously been subjected to a forced swim test. Pre-exposure of nonenriched animals to a Porsolt test (the so-called initial Porsolt; CPP group) induced a significant notable increase in the immobility time in the final Porsolt. Relevantly, the score of the pre-exposed enriched animals (EPP) did not improve in the final Porsolt, as the EE had no effect on decreasing the immobility time, which was indistinguishable from that of pre-exposed non-enriched mice (CPP).

After sacrificing the animals, we analyzed a battery of parameters in the brain to determine how neurogenic populations responded to the manipulations. We found significant differences in the number of the older new neurons, those labeled with CldU at the beginning of the experiment (3-month-old new neurons; Figure 3a-d; twoway ANOVA for housing and Porsolt; ANOVA $\mathrm{F}_{3,27}$ 5.467, $p<0.05$. F $F_{\text {housing } 1,27} 12.081, p=0.001 ; \mathrm{F}_{\text {Porsolt 1,27 }} 2.514$, $p=0.124 ; \mathrm{F}_{\text {housing }} \times$ Porsolt $\left.1,270.569, p=0.457\right)$. The number of these neurons increased after EE when compared with non-enriched animals, significantly in animals suffering the

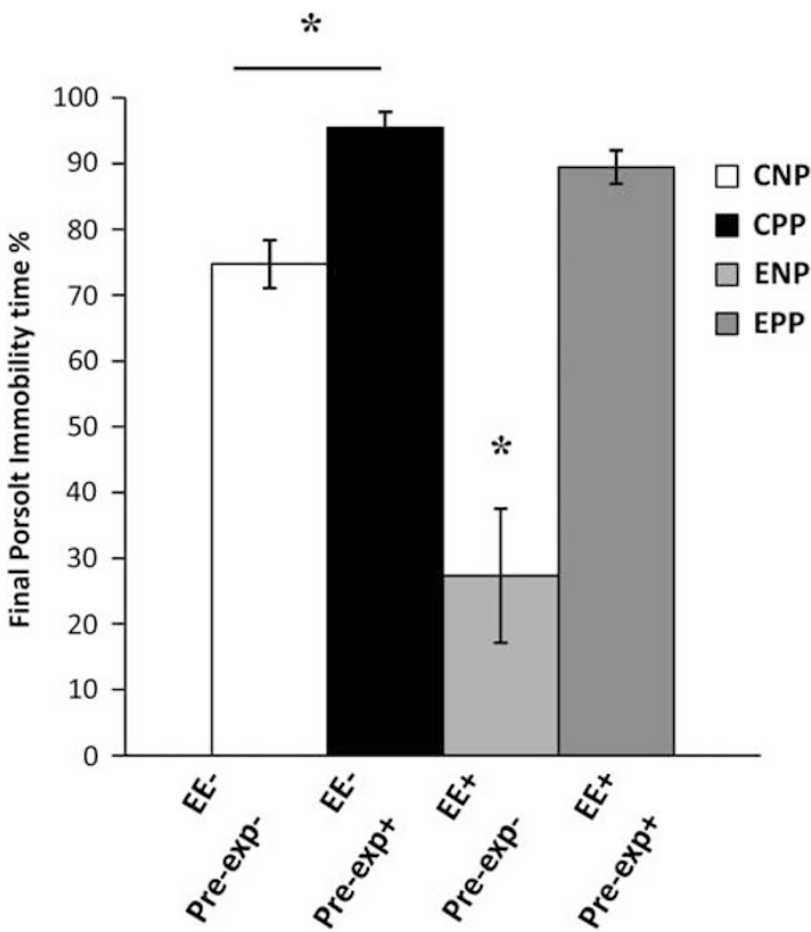

Figure 2 Final Porsolt test. Behavioral despair was evaluated by means of a learned helplessness paradigm consisting of a stressful forced swim test. The chart represents the percentage time the animals were immobile floating with only slight movements to keep breathing (a common measure of mood status). Animals pre-exposed to the same type of Porsolt test at the beginning of the experiment spent more time immobile (CPP), while those living in an enriched environment significantly decreased the immobility (ENP). The enrichment was not able to recover the effect of the initial Porsolt when animals were tested in the second, final Porsolt (EPP). $C N P$, controls $N=9$; $C P P, N=10 ; E N P, N=9$; $E P P, N=10$. ${ }^{*} P<0.005$. final Porsolt and as a tendency in the animals suffering two Porsolt tests (the initial and final tests; Figure 5a). Interestingly, pre-exposed non-enriched mice (CPP) had a similar number of $\mathrm{CldU}^{+}, 3$-month-old new neurons than controls (CNP). It is interesting to note that we found no $\mathrm{CldU}^{+}$cells co-labeled with DCX, as expected and corresponds to 3-month-old new neurons. By contrast, the younger new neurons, those labeled with IdU close to the end of the experiment (1-week-old new neurons; Figure $3 \mathrm{e}-\mathrm{h}$ ) significantly decreased in number (CPP) when compared with controls (CNP) as a consequence of suffering two Porsolts (initial and final; Figure 5b). EE did

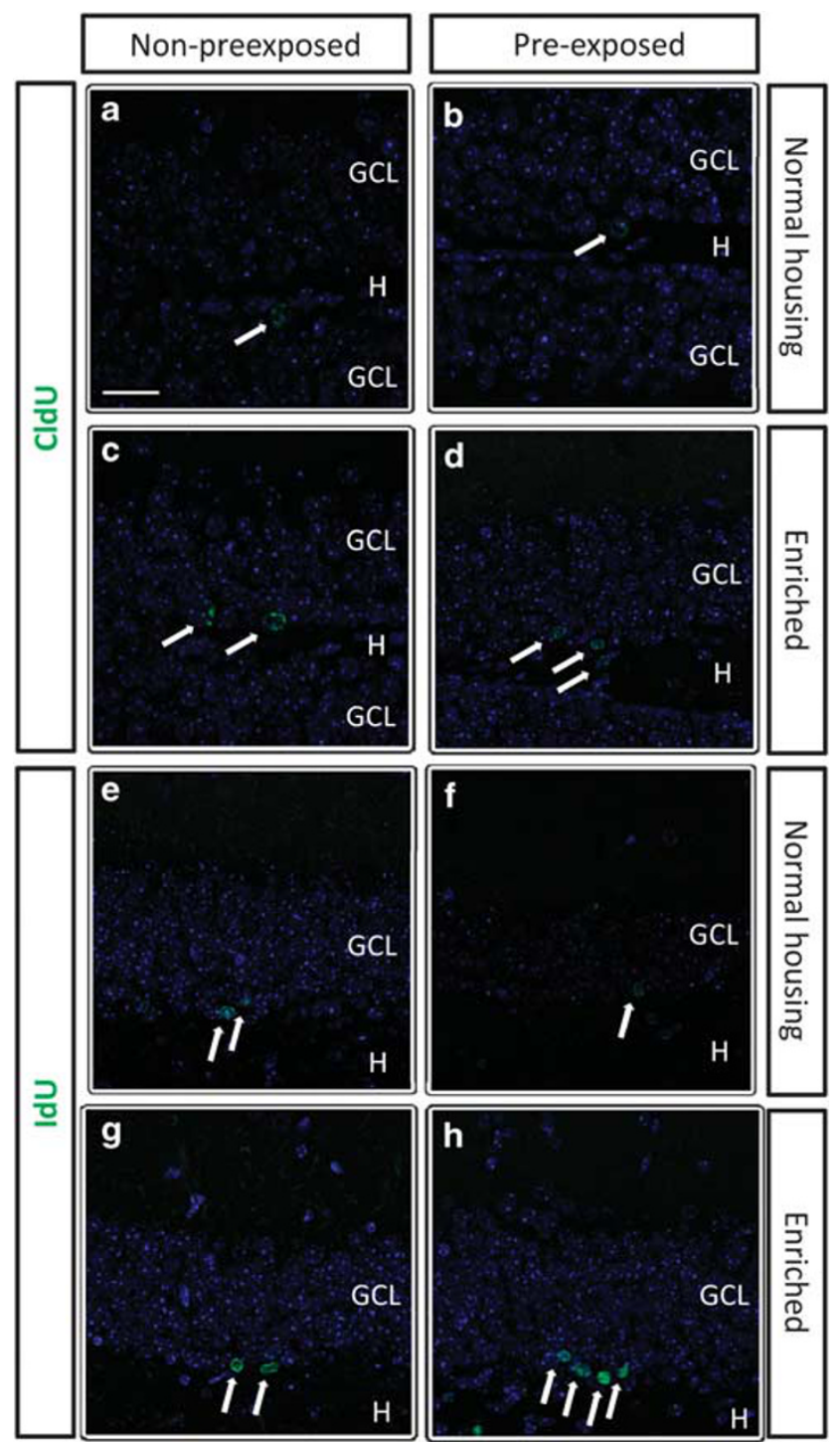

Figure 3 Representative images of the immature new neurons labeled with CldU (a-d) or IdU (e-h). Naive non-pre-exposed animals (a, c, e, g) were only exposed to the final Porsolt test, while pre-exposed animals (b, d, f, h) were also exposed to an initial Porsolt at the beginning of the experiment. The pictures show the granule cell layer of the dentate gyrus at a rostral level (coronal sections). Positive cells (arrows) are mainly located in the subgranular zone or in the inner part of the granule layer. The animals living in an enriched environment for 10 weeks (c, d, g, h) had more new neurons than control, normally housed animals ( $a, b, e, f)$, both 3-monthold (CldU; a-d) and I-week-old cells (IdU; e-h). GCL, granule cell layer; $H$, hilus. Scale bar, $40 \mu \mathrm{m}$. 
not change the number of these young, 1-week-old new neurons (ENP) when compared with control animals (CNP), while in pre-exposed enriched animals (EPP), we found a significantly increased number of these cells compared with controls and non-pre-exposed enriched animals (Figure 5b; two-way ANOVA for housing and Porsolt; ANOVA $\mathrm{F}_{3,23}$ 12.157, $p<0.0001$. F housing $1,23_{14.241,} p=0.001 ; F_{\text {Porsolt } 1,23}$ $0.034, p=0.856 ; \quad F_{\text {housing }} \times$ Porsolt 1,23 17.617, $\left.p=0.0001\right)$. Additionally, we have found that $99 \%$ of $\mathrm{IdU}^{+}$cells are also $\mathrm{DCX}^{+}$, as expected considering the age of the $\mathrm{IdU}^{+}$cells.

To determine how these changes might be influenced by variations in cell proliferation or cell death, we measured proliferation by means of $\mathrm{pH}^{+}$or $\mathrm{Ki}^{+} 7^{+}$cell counting and cell death by means of fractin ${ }^{+}$cell counting. We have found that the different manipulations did not significantly influence the number of fractin ${ }^{+}$cells in any experimental group (two-way ANOVA for housing and Porsolt; ANOVA $\mathrm{F}_{3,28} 0.738, p<0.538$. $\mathrm{F}_{\text {housing } 1,28} 0.976, p=0.332 ; \mathrm{F}_{\text {Porsolt } 1,28}$ $\left.0.594, \quad p=0.447 ; \quad F_{\text {housing } \times \text { Porsolt } 1,28} \quad 0.643, \quad p=0.429\right)$. Likewise, there were differences neither in the number of $\mathrm{pH}^{+}$cells at the time of kill (two-way ANOVA for housing and Porsolt; ANOVA $\mathrm{F}_{3,28} 1.633, p<0.204$. F $_{\text {housing } 1,28} 0.565$, $p=0.459 ; \mathrm{F}_{\text {Porsolt } 1,28} 2.830, p=0.104 ; \mathrm{F}_{\text {housing } \times \text { Porsolt } 1,28}$ $1.504, p=0.23$ ), nor in the number of $\mathrm{Ki}^{+}$cells (two-way ANOVA for housing and Porsolt; ANOVA $\mathrm{F}_{3,28}$ 1.581, $p<0.316$. $F_{\text {housing 1,28 }} 0.511, p=0.482 ; F_{\text {Porsolt 1,28 }} 2.614$, $p=0.136 ; F_{\text {housing }} \times$ Porsolt $\left.1,281.566, p=0.332\right)$.

To analyze the impact of the different manipulations on the immature neurons according to their state of differentiation, we measured the survival of two distinct cell subpopulations (Figure 4), immature neurons with a proliferative capacity ( $\mathrm{DCX}^{+} / \mathrm{Calret}^{-}$cells) and postmitotic immature neurons $\left(\mathrm{DCX}^{+} / \mathrm{Calret}^{+}\right.$cells). We found significant differences in the total number of both subpopulations $\left(\mathrm{DCX}^{+} / \mathrm{Calret}^{-}\right.$cells: two-way ANOVA for housing and Porsolt; ANOVA $\mathrm{F}_{3,28}$ 6.600, $p<0.002$. $\mathrm{F}_{\text {housing } 1,28}$ 6.766, $p=0.015 ; \mathrm{F}_{\text {Porsolt 1,28 }}$ 7.785, $p=0.009$; $\mathrm{F}_{\text {housing }} \times$ Porsolt 1,28 $5.250, p=0.03$ DCX $^{+} /$Calret $^{+}$cells: twoway ANOVA for housing and Porsolt; ANOVA $\mathrm{F}_{3,25} 8.322$, $p<0.001$. F Fousing 1,25 23.674, $p=0.0001$; F Forsolt 1,25 0.140, $\left.p=0.712 ; F_{\text {housing } \times \text { Porsolt } 1,25} 0.798, p=0.38\right)$. We found that immature neurons, both cells with proliferative ability and postmitotic, were significantly increased after EE (ENP; Figure $5 c$ and d). However, only postmitotic $\mathrm{DCX}^{+} / \mathrm{Calret}^{+}$ neurons augmented in animals subjected to two Porsolt tests (initial and final, EPP; Figure 5d). In these animals, $\mathrm{DCX}^{+} /$Calret $^{-}$cells remained unaltered after manipulation (Figure 5c).

It is noteworthy that the total volume of the granule cell layer of the dentate gyruys was not significantly different in any of the experimental groups (two-way ANOVA for housing and Porsolt; ANOVA $\mathrm{F}_{3,28}$ 1.203, $p<0.327$. $\mathrm{F}_{\text {housing } 1,28} 1.319, p=0.261 ; \mathrm{F}_{\text {Porsolt 1,28 }} 0.229, \quad p=0.636$; $\mathrm{F}_{\text {housing }} \times$ Porsolt 1,28 2.060, $p=0.162$ ) nor did the total number of granule neurons in the granule cell layer (twoway ANOVA for housing and Porsolt; ANOVA F 3,28 1.732, $p<0.183$. F Fousing 1,28 1.154, $p=0.292 ; \mathrm{F}_{\text {Porsolt 1,28 }} 0.279$, $p=0.602 ; \mathrm{F}_{\text {housing }} \times$ Porsolt $\left.1,283.763, p=0.063\right)$. To analyze the impact of the treatments on the activation of granule neurons, we measured the number of $\mathrm{cfos}^{+}$cells in the granule cell layer. We found no significant differences

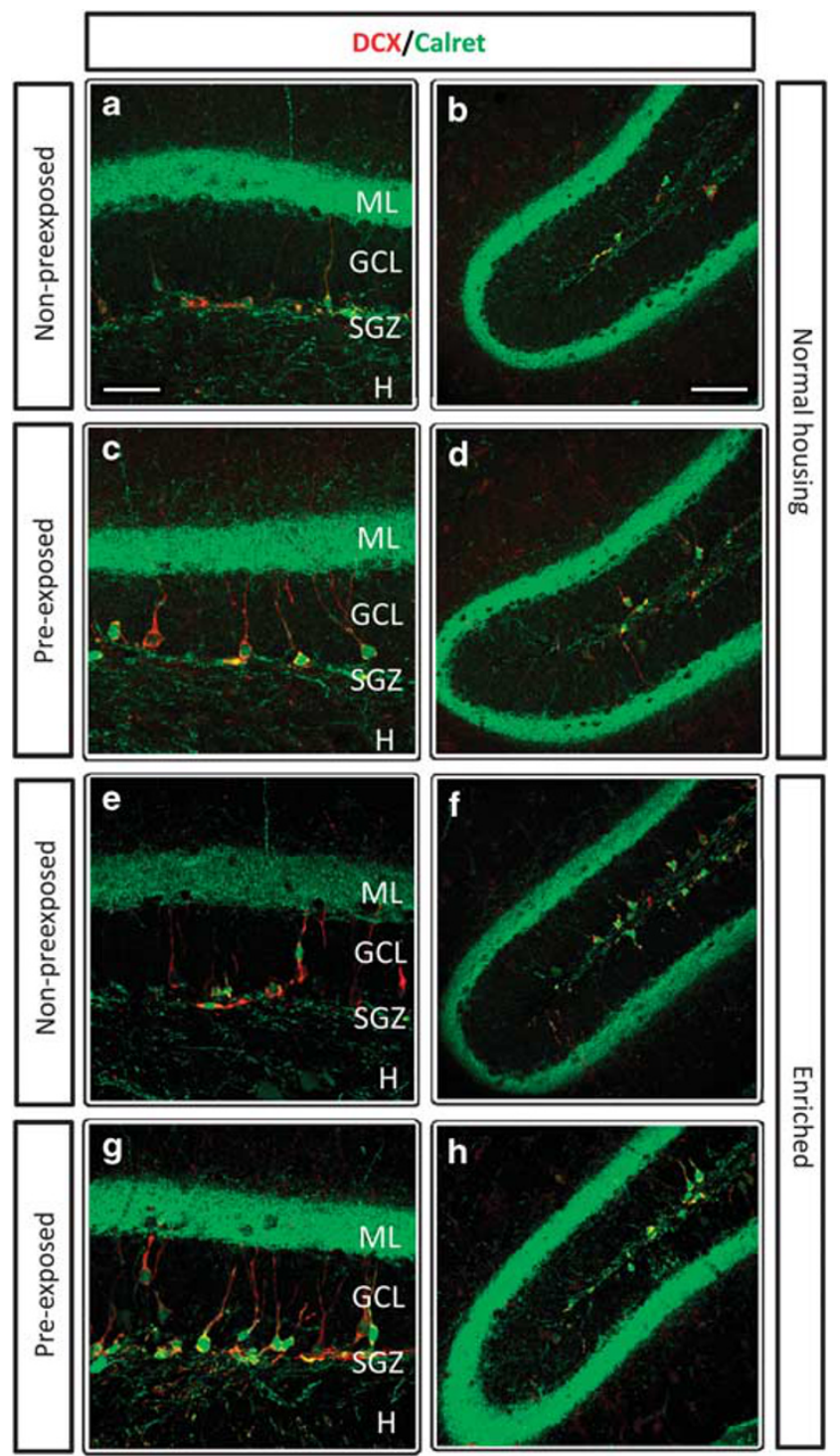

Figure 4 Representative images of the immature new neurons labeled with DCX (red) and/or Calret (green). The pictures represent the granule cell layer, suprapyramidal blade, of the dentate gyrus at a rostral level (coronal sections). Animals maintained in normal housing conditions (a-d) had a normal distribution of these subpopulations of immature neurons, while animals living in an enriched environment ( $\mathrm{e}-\mathrm{h})$ had more immature neurons. ML, molecular layer; GCL, granule cell layer; SGZ, subgranular zone; $\mathrm{H}$, hilus. Scale bars, a (for a, c, e and g) $50 \mu \mathrm{m}$; b (for b, d, $f$ and h) $100 \mu \mathrm{m}$.

between groups (two-way ANOVA for housing and Porsolt; ANOVA $F_{3,22}$ 1.631, $p<0.211$. F $F_{\text {housing } 1,22} 0.345, p=0.563$; $\mathrm{F}_{\text {Porsolt 1,22 }} \quad 4.809, \quad p=0.039 ; \quad \mathrm{F}_{\text {housing } \times \text { Porsolt 1, 22 }} \quad 0.050$, $p=0.826)$; however, there was a tendency for a higher number of $\mathrm{cfos}^{+}$cells to accumulate in the non-enriched pre-exposed animals (CPP) when compared with the rest of the groups, as expected and previously reported (Sibille et al, 1997), accordingly to the rapid response of the tissue to the exacerbated effects of stress. There were no significant intragroup correlations between the number of $\mathrm{Cldu}^{+}$and $\mathrm{IdU}^{+}$cells (Pearson correlation ${ }_{\mathrm{CNP}}-0.404$, $p=0.5$; Pearson $_{\mathrm{CPP}}-0.242, p=0.564 ;$ Pearson $_{\mathrm{EPP}} 0.640$, $p=0.122)$, except for the enriched non-pre-exposed animals (Pearson $_{\mathrm{ENP}}$ 0.893, $p=0.017$ ). 

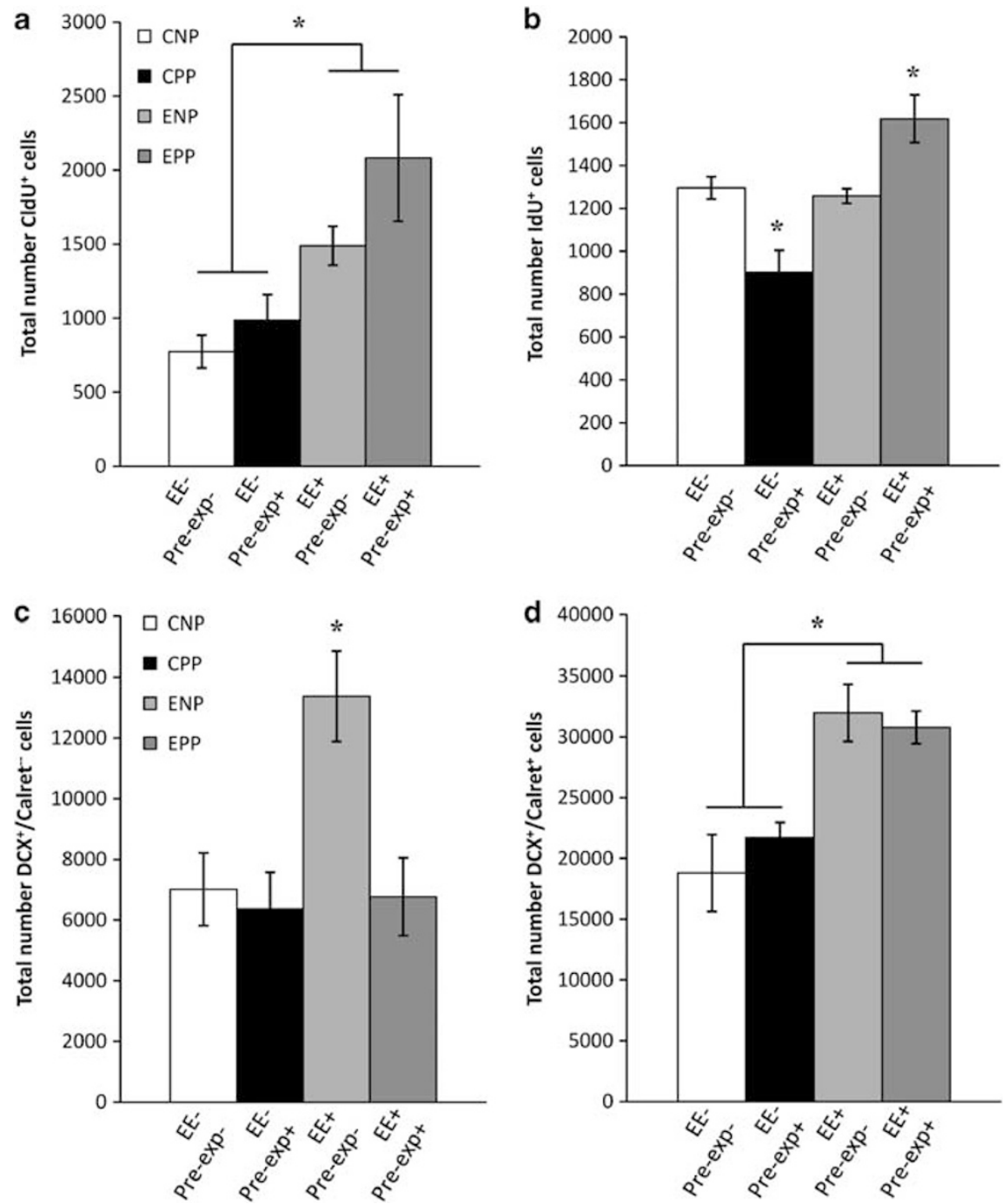

Figure 5 Effects of pre-exposure to the same stressor and EE on the number of new neurons measured either by thymidine analogs injections (CldU; 3-month-old cells, a; IdU, I-week-old cells, b) or by the state of differentiation assessed by dual immunohistochemistry by with DCX and Calret, to distinguish immature neurons in distinct states of differentiation (DCX ${ }^{+} /$Calret $^{-}$neurons with proliferative ability, c; or postmitotic DCX ${ }^{+} / C^{2}$ alret ${ }^{+}$neurons, d). EE significantly increased the number of 3-month-old new neurons $\left(\mathrm{CldU}^{+}\right.$, a). Younger I-week-old new neurons $\left(\mathrm{IdU}^{+}, \mathrm{b}\right)$ were sensitive both to pre-exposure to the stressor and to the sum of enrichment and pre-exposure. $\mathrm{DCX}^{+} / \mathrm{Calret}^{-}$cells were increased after enrichment but this effect was only achieved in naive animals that were not pre-exposed to the stressor (c), while $\mathrm{DCX}^{+} / \mathrm{Calret}^{+}$cells increased independently of the pre-exposure factor (d). *P $<0.01$.

\section{DISCUSSION}

In order to determine whether the antidepressant and proneurogenic effects of EE differ in naive or pre-exposed animals in response to an stressor, and whether this has distinct effects on different neurogenic subpopulations, we have exposed animals to a Porsolt test (at the end of the experiment, consequently called final Porsolt) in one of four distinct situations: animals lived either enriched or control housed during 10 weeks, and besides, animals were either pre-exposed or not to a Porsolt test before the enrichment (at the beginning of the experiment, consequently called initial Porsolt).

We first analyzed whether the antidepressant effect of EE, as measured in a Porsolt test of forced swimming (learned helplessness), is distinct in naive animals when compared with animals pre-exposed to the same Porsolt test before the start of a long time period of EE. As expected (Laviola et al, 2008; Llorens-Martin et al, 2007; Porsolt et al, 1978), EE had an antidepressant effect that could be measured by the reduction in the immobility time. Pre-exposure to the same stressor (the initial Porsolt test) in control non-enriched animals led to an exacerbated effect in the second (final) Porsolt test, reflected as an increase in the immobility time. This long-term (10 weeks) effect is similar to that reported over shorter periods immediately before the test or with pretest sessions (Borsini et al, 1989), as well as when studying different parameters like corticosterone levels (Murison et al, 1986), or the effects of antidepressant drugs (Armario et al, 1988; Borsini et al, 1989; Hilakivi et al, 1989). This action is not surprising, as different stressors have been shown inducing pre-exposure-induced response sensitization to similar, less intense stressors (Rau et al, 2005).

These results raise an important issue. It is possible that the animals remember this kind of highly stressful stimulus (initial Porsolt) for 10 weeks during their adult life and that the 'negative' effects of this initial stimulus can be 
exacerbated after this time (as observed in the final Porsolt). This, in turn, is possible because the time course of the enhanced response to a stressor pre-exposure has been analyzed in certain paradigms, finding that enhancement persists for at least 3 months, at least in the fear conditioning paradigm (Rau and Fanselow, 2009). Alternatively, it is possible that the animals performed the final Porsolt with an exacerbated response as the consequence of having been exposed to any stressful stimulus, not necessarily the same stimulus. Both of these possibilities have been demonstrated previously (Rau and Fanselow, 2009; Sabban and Serova, 2007). We addressed this question by including enrichment in the process, because it is not clear whether EE sensitizes the brain to novelty (as it is a paradigm of continuously renovated novelty, increasing the ability to cope with further stressors (Fox et al, 2006)), or alternatively, it desensitizes the brain to novelty, so that the animal might develop a higher threshold to the perception of threat in the environment. This effect might mean the animal learns helplessness paradigms over a different temporal scale, the behavioral correlate of the antidepressant effect of EE. To this end, animals were subjected to an EE before the Porsolt test, both control animals, and animals with a homotypic pre-exposure to the same stressor (Porsolt test), since pre-exposure to forced swimming may produce sensitization (lower threshold) to subsequent exposures (see above). Our results show that the effect of an $\mathrm{EE}$ is not dependent on any desensitization to novelty and indeed, EE had no effect in pre-exposed animals.

An important result is that the antidepressant effect of EE required naive animals to take place. Pre-exposed animals living at an enriched environment were not able to decrease their scores in the final Porsolt test. Furthermore, the preexposed enriched animals displayed scores indistinguishable from those of the pre-exposed non-enriched mice, that is, significantly different than those of the control animals (naive non-enriched). This result points to different pathways for the actions of stressful stimulus and enriched physical-cognitive activity, both aspects inducing independent, summing effects in displaying the behavioral despair (in the present experimental design, both aspects are opposite, and pre-exposure to forced swim being predominant); nevertheless, the result do not let to discard the possibility that the different pathways could be influencing the same final target (ie either glucocorticoids or IGF1, eg, acting on the same neuronal cohort).

In this context, the analysis of changes in the brain (adult hippocampal neurogenesis) acquires special importance. For this reason, we analyzed whether this experimental model had distinct effects on the neurogenic subpopulations with different stages of cell differentiation. On the one hand, we measured the total number of 3-month-old cells compared with the number of 1-week-old cells in the granule cell layer of the dentate gyrus. On the other hand, we measured the total number of $\mathrm{DCX}^{+} /$Calret $^{-}$immature neurons with proliferative capacity compared with the number of postmitotic $\mathrm{DCX}^{+} /$Calret $^{+}$neurons.

The 10-week EE had a distinct effect on subpopulations of different age, as expected: EE increased the total number of older new neurons, those labeled with CldU at the beginning of the experiment (3-month-old new neurons), while it had no significant effect on the total number of the younger new neurons, those labeled with IdU towards the end of the experiment (1-week-old new neurons), as expected and previously described in the literature (Kempermann et al, 1997b; Llorens-Martin et al, 2007, 2010). Likewise, preexposure to the stressful event (initial Porsolt) had specific effects on the cells of different ages: whereas pre-exposure had no effect on the number of 3-month-old new neurons $\left(\mathrm{CldU}^{+}\right)$, it significantly decreased the number of 1-weekold new neurons $\left(\mathrm{IdU}^{+}\right)$. Therefore, the older new neurons are both sensitive to the long-term effects of enrichment, and resistant to pre-exposure and further exposure to the stressor. By contrast, the younger new neurons do not respond to enrichment over a long time period (see also Llorens-Martin et al, 2010), yet they are sensitive to the stressful stimulus (these younger cells are also sensitive to a single stressful event as demonstrated elsewhere (Llorens-Martin et al, 2007).

Interestingly, the number of immature neurons increased after pre-exposure and enrichment together (what was a tendency in the older new neurons represented a significant difference in the younger new neurons). This effect may be explained as the sum of a compensatory effect on long-term survival plus the action of enrichment. It should be borne in mind that the initial Porsolt test (pre-exposure) could well have induced an initial increase in cell death in young immature neurons (those CldU labeled at the beginning of the experiment; this has been demonstrated elsewhere (Llorens-Martin et al, 2007). This initially increased cell death has been compensated along the next 10 weeks of normal housing (CPP group, present results). This compensation is robust, as the remaining cells are able to resist the exposure to the second (final) Porsolt test. Therefore, we hypothesize that cell survival may increase in the dentate gyrus as a compensatory mechanism after stress over a long time period (even in normal housing conditions), and thus, the additive effect of an enriched environment (EPP group) could further increase the survival of more neurons. This explanation would also be valid for the increased number of 1-week-old new neurons. However, in this case, the superimposed effect would influence short-term, more than long-term survival, due to the short period between labeling and kill. Thus, while the cell death induced by the initial Porsolt test in normally housed animals may be compensated for a potent increase in long-term cell survival, this increase could be exacerbated further in enriched animals $\left(\mathrm{CldU}^{+}\right.$cells). By contrast, short-term cell survival ( $\mathrm{IdU}^{+}$ cells) responds in a completely different way to that previously published about the survival under enrichment conditions (Kempermann et al, 1997b; Llorens-Martin et al, 2007, 2010): we have found evidences of response in cell proliferation neither in naive enriched (ENP group) nor in pre-exposed normal housed animals (CPP group); therefore, given the initial cell death and subsequent exacerbated long-term increase in $\mathrm{CldU}^{+}$cell survival due to EE, $\mathrm{IdU}^{+}$ short-term survival may also be increased (EPP group). The lack of significant variation in the number of $\mathrm{pH}^{+}$cells points to the effects described here as the result of the changes in cell survival. Changes in cell proliferation alone does not account for the results obtained here, as the percentage of young immature neurons (1-week-old $\mathrm{IdU}^{+}$ cells) co-expressing $\mathrm{pH} 3$ is only $\approx 3 \%$ (results not shown). Nevertheless, an increase in cell proliferation might still 
account for some of the effect on the $\mathrm{CldU}^{+}$cell numbers, together with an increase in long-term survival.

Moreover, the 10-week EE increased the total number of immature neurons irrespective of their state of differentiation. Accordingly, the number of both $\mathrm{DCX}^{+} /$Calret $^{-}$and postmitotic $\mathrm{DCX}^{+} /$Calret $^{+}$neurons increased significantly after enrichment (similar results have been obtained by different works; Komitova et al, 2005; Llorens-Martin et al, 2007, 2010). By contrast, pre-exposure to the stressor had distinct effects on these subpopulations in different states of maturation, as hypothesized: while postmitotic $\mathrm{DCX}^{+} / \mathrm{Calret}^{+}$neurons does not respond to the preexposure and further exposure to the stressor, the final Porsolt test prevented the EE-induced increase in the number of more immature $\mathrm{DCX}^{+} /$Calret $^{-}$neurons (we suggest that the most immature neurons are highly sensitive to the stressor when there are many such neurons). The subpopulation of the most immature neurons analyzed, $\mathrm{DCX}^{+} /$Calret $^{-}$cells, is the more sensitive of the immature new neurons, as also shown after the sudden cessation of a long-term enrichment (Llorens-Martin et al, 2010).

By comparing the data from the forced swimming test and that from the immature neurogenic subpopulations, we suggest that the significant changes in these cell populations, consistent with changes in behavior are those of $\mathrm{IdU}^{+}, 1$-week-old cells, and $\mathrm{DCX}^{+} /$Calret $^{-}$immature neurons; therefore, we conclude that a close relationship may exist between the behavior analyzed here and the youngest immature neurons in the dentate gyrus population, a relationship that will be interesting to explore further. Our belief that the treatments had a distinct effect on different subpopulations of immature neurons is supported by the fact that EE had a protective influence when examining the effect of pre-exposure to the reexposition to the same stressor, rather than influencing recovery from the effects of the initial exposure. This is also valid for behavior, in contrast to what has been described for different stressors such as social conflict (Schloesser et al, 2010) or in mouse models of increased anxiety/ depression such as partial BDNF deletion (Chourbaji et al, 2008); as exposed in our working hypothesis, the extinguishing effect of enrichment on the response to an adverse stimulus depends entirely on the stressor. Besides, there is a differential effect of the treatment on distinct neurogenic subpopulations distinguished by age/state of differentiation. Hence, younger cells are more sensitive and responsive to the conditions of the environment, both positive and negative stimuli. Older cells are probably better integrated into a circuit and thus, they are more prone to resist manipulation. Whatever the case, a direct relationship between neurogenesis and the behavioral effects described here will need further investigation and more direct evidences to be firmly established.

These results are relevant to identify cell populations that are targets of stress, depression, and enrichment as a part of the mechanism responsible for mood dysfunctions. In addition, better understanding the long-term effects of antidepressant interventions like EE might be of growing interest when considered during puberty/adolescence, especially if effective therapies can be developed against stress during adult life.

\section{ACKNOWLEDGEMENTS}

We thank Belén García and Carmen Hernández for their help with the confocal microscopy, and Laude Garmendia for her help with the animals at the Cajal Institute. We also appreciate the assistance of Dr Mark Sefton in revising the manuscript. Grant awarded to JLT from the Ministerio de Educacion y Ciencia. Grant number: BFU2007-60195/BFI. The funders had no role in study design, data collection and analysis, decision to publish, or preparation of the manuscript.

\section{DISCLOSURE}

The authors declare that, except for income received from the primary employer, no financial support or compensation has been received from any individual or corporate entity over the past 3 years for research or professional service and there are no personal financial holdings that could be perceived as constituting a potential conflict of interest.

\section{REFERENCES}

Armario A, Gavalda A, Marti O (1988). Forced swimming test in rats: effect of desipramine administration and the period of exposure to the test on struggling behavior, swimming, immobility and defecation rate. Eur J Pharmacol 158: 207-212.

Borsini F, Lecci A, Sessarego A, Frassine R, Meli A (1989). Discovery of antidepressant activity by forced swimming test may depend on pre-exposure of rats to a stressful situation. Psychopharmacology (Berl) 97: 183-188.

Cordoba F, Yusta B, Munoz-Blanco J (1984). Changes in neurotransmitter amino acids and protein in CNS areas of mice subjected to differential housing conditions. Pharmacol Biochem Behav 21: 349-352.

Chourbaji S, Brandwein C, Vogt MA, Dormann C, Hellweg R, Gass $P$ (2008). Nature vs. nurture: can enrichment rescue the behavioural phenotype of BDNF heterozygous mice? Behav Brain Res 192: 254-258.

Christie BR, Eadie BD, Kannangara TS, Robillard JM, Shin J, Titterness AK (2008). Exercising our brains: how physical activity impacts synaptic plasticity in the dentate gyrus. Neuromolecular Med 10: 47-58.

Detke MJ, Rickels M, Lucki I (1995). Active behaviors in the rat forced swimming test differentially produced by serotonergic and noradrenergic antidepressants. Psychopharmacology (Berl) 121: 66-72.

Dupret D, Revest JM, Koehl M, Ichas F, De Giorgi F, Costet P et al (2008). Spatial relational memory requires hippocampal adult neurogenesis. PLoS One 3: e1959.

Fox C, Merali Z, Harrison C (2006). Therapeutic and protective effect of environmental enrichment against psychogenic and neurogenic stress. Behav Brain Res 175: 1-8.

García-Segura LM (2009). Hormones and Brain Plasticity. Oxford University Press: Oxford.

Greer TL, Trivedi MH (2009). Exercise in the treatment of depression. Curr Psychiatry Rep 11: 466-472.

Hilakivi LA, Durcan MJ, Lister RG (1989). Effects of ethanol on fight- or swim-stressed mice in Porsolt's swim test. Neuropsychopharmacology 2: 293-298.

Hutchinson E, Avery A, Vandewoude S (2005). Environmental enrichment for laboratory rodents. ILAR J 46: 148-161.

Kempermann G (2008). The neurogenic reserve hypothesis: what is adult hippocampal neurogenesis good for? Trends Neurosci 31: 163-169. 
Kempermann G, Kuhn HG, Gage FH (1997a). Genetic influence on neurogenesis in the dentate gyrus of adult mice. Proc Natl Acad Sci USA 94: 10409-10414.

Kempermann G, Kuhn HG, Gage FH (1997b). More hippocampal neurons in adult mice living in an enriched environment. Nature 386: 493-495.

Kempermann G, Kuhn HG, Gage FH (1998). Experience-induced neurogenesis in the senescent dentate gyrus. J Neurosci 18: 3206-3212.

Komitova M, Mattsson B, Johansson BB, Eriksson PS (2005). Enriched environment increases neural stem/progenitor cell proliferation and neurogenesis in the subventricular zone of stroke-lesioned adult rats. Stroke 36: 1278-1282.

Laviola G, Hannan AJ, Macri S, Solinas M, Jaber M (2008). Effects of enriched environment on animal models of neurodegenerative diseases and psychiatric disorders. Neurobiol Dis 31: 159-168.

Leal-Galicia P, Saldivar-Gonzalez A, Morimoto S, Arias C (2007). Exposure to environmental enrichment elicits differential hippocampal cell proliferation: role of individual responsiveness to anxiety. Dev Neurobiol 67: 395-405.

Leuner B, Glasper ER, Gould E (2009). Thymidine analog methods for studies of adult neurogenesis are not equally sensitive. J Comp Neurol 517: 123-133.

Llorens-Martin M, Torres-Aleman I, Trejo JL (2006). Pronounced individual variation in the response to the stimulatory action of exercise on immature hippocampal neurons. Hippocampus 16: 480-490.

Llorens-Martin M, Torres-Aleman I, Trejo JL (2009). Mechanisms mediating brain plasticity: IGF1 and adult hippocampal neurogenesis. Neuroscientist 15: 134-148.

Llorens-Martin MV, Rueda N, Martinez-Cue C, Torres-Aleman I, Florez J, Trejo JL (2007). Both increases in immature dentate neuron number and decreases of immobility time in the forced swim test occurred in parallel after environmental enrichment of mice. Neuroscience 147: 631-638.

Llorens-Martin M, Tejeda GS, Trejo JL (2010). Differential regulation of the variations induced by environmental richness in adult neurogenesis as a function of time. A dual birthdating analysis. PLoS One 5: e12188.

Mandolesi L, De Bartolo P, Foti F, Gelfo F, Federico F, Leggio MG et al (2008). Environmental enrichment provides a cognitive reserve to be spent in the case of brain lesion. J Alzheimers Dis 15: $11-28$.

Martinez-Cue C, Baamonde C, Lumbreras M, Paz J, Davisson MT, Schmidt C et al (2002). Differential effects of environmental enrichment on behavior and learning of male and female Ts65Dn mice, a model for Down syndrome. Behav Brain Res 134: 185-200.

Meshi D, Drew MR, Saxe M, Ansorge MS, David D, Santarelli L et al (2006). Hippocampal neurogenesis is not required for behavioral effects of environmental enrichment. Nat Neurosci 9: 729-731.

Milgram NW, Siwak-Tapp CT, Araujo J, Head E (2006). Neuroprotective effects of cognitive enrichment. Ageing Res Rev 5: 354-369.

Mora F, Segovia G, del Arco A (2007). Aging, plasticity and environmental enrichment: structural changes and neurotransmitter dynamics in several areas of the brain. Brain Res Rev 55: 78-88.

Murison R, Overmier JB, Skoglund EJ (1986). Serial stressors: prior exposure to a stressor modulates its later effectiveness on gastric ulceration and corticosterone release. Behav Neural Biol 45: 185-195.

Navailles S, Hof PR, Schmauss C (2008). Antidepressant drug-induced stimulation of mouse hippocampal neurogenesis is age-dependent and altered by early life stress. J Comp Neurol 509: 372-381.

Nithianantharajah J, Hannan AJ (2009). The neurobiology of brain and cognitive reserve: mental and physical activity as modulators of brain disorders. Prog Neurobiol 89: 369-382.

Olson AK, Eadie BD, Ernst C, Christie BR (2006). Environmental enrichment and voluntary exercise massively increase neurogenesis in the adult hippocampus via dissociable pathways. Hippocampus 16: 250-260.

Petrosini L, De Bartolo P, Foti F, Gelfo F, Cutuli D, Leggio MG et al (2009). On whether the environmental enrichment may provide cognitive and brain reserves. Brain Res Rev 61: 221-239.

Porsolt RD, Anton G, Blavet N, Jalfre M (1978). Behavioural despair in rats: a new model sensitive to antidepressant treatments. Eur J Pharmacol 47: 379-391.

Porsolt RD, Bertin A, Jalfre M (1977a). Behavioral despair in mice: a primary screening test for antidepressants. Arch Int Pharmacodyn Ther 229: 327-336.

Porsolt RD, Le Pichon M, Jalfre M (1977b). Depression: a new animal model sensitive to antidepressant treatments. Nature 266: 730-732.

Rau V, DeCola JP, Fanselow MS (2005). Stress-induced enhancement of fear learning: an animal model of posttraumatic stress disorder. Neurosci Biobehav Rev 29: 1207-1223.

Rau V, Fanselow MS (2009). Exposure to a stressor produces a long lasting enhancement of fear learning in rats. Stress 12: 125-133.

Sabban EL, Serova LI (2007). Influence of prior experience with homotypic or heterotypic stressor on stress reactivity in catecholaminergic systems. Stress 10: 137-143.

Sale A, Berardi N, Maffei L (2009). Enrich the environment to empower the brain. Trends Neurosci 32: 233-239.

Schaeffer EL, Novaes BA, da Silva ER, Skaf HD, Mendes-Neto AG (2009). Strategies to promote differentiation of newborn neurons into mature functional cells in Alzheimer brain. Prog Neuropsychopharmacol Biol Psychiatry 33: 1087-1102.

Schloesser RJ, Lehmann M, Martinowich K, Manji HK, Herkenham M (2010). Environmental enrichment requires adult neurogenesis to facilitate the recovery from psychosocial stress. Mol Psychiatry 15: 1152-1163.

Segovia G, Yague AG, Garcia-Verdugo JM, Mora F (2006). Environmental enrichment promotes neurogenesis and changes the extracellular concentrations of glutamate and GABA in the hippocampus of aged rats. Brain Res Bull 70: 8-14.

Sibille E, Sarnyai Z, Benjamin D, Gal J, Baker H, Toth M (1997). Antisense inhibition of 5-hydroxytryptamine2a receptor induces an antidepressant-like effect in mice. Mol Pharmacol 52: 1056-1063.

Stead JD, Clinton S, Neal C, Schneider J, Jama A, Miller S et al (2006). Selective breeding for divergence in novelty-seeking traits: heritability and enrichment in spontaneous anxietyrelated behaviors. Behav Genet 36: 697-712.

Sztainberg Y, Kuperman Y, Tsoory M, Lebow M, Chen A (2010). The anxiolytic effect of environmental enrichment is mediated via amygdalar CRF receptor type 1. Mol Psychiatry 15: 905-917.

Trejo JL, Llorens-Martin MV, Torres-Aleman I (2008). The effects of exercise on spatial learning and anxiety-like behavior are mediated by an IGF-I-dependent mechanism related to hippocampal neurogenesis. Mol Cell Neurosci 37: 402-411.

Trejo JL, Piriz J, Llorens-Martin MV, Fernandez AM, Bolos M, LeRoith D et al (2007). Central actions of liver-derived insulinlike growth factor I underlying its pro-cognitive effects. Mol Psychiatry 12: 1118-1128.

van Praag H, Kempermann G, Gage FH (1999). Running increases cell proliferation and neurogenesis in the adult mouse dentate gyrus. Nat Neurosci 2: 266-270. 